\title{
Escala de Avaliação das Percepções sobre o Programa Mais Médicos
}

\author{
Cynthia de Freitas Melo - Universidade de Fortaleza, Fortaleza, Brasil \\ Mariana Carvalho Costa - Universidade de Fortaleza, Fortaleza, Brasil \\ Bárbara Jéssyca Magalhães - Universidade de Fortaleza, Fortaleza, Brasil
}

\begin{abstract}
Resumo
A presente pesquisa objetivou criar um instrumento de avaliação da eficácia do Programa Mais Médicos (PMM), apreendendo as percepções de uma amostra não probabilística nacional estratificada de 643 brasileiros. Foi realizada análise fatorial na escala, e os dados de avaliação do PMM foram analisados por meio de estatística descritiva, bivariada e multivariada. Com indicação de melhores valores psicométricos e explicação teórica, o método Componentes Principais, com rotação varimax, estruturou os 35 itens da escala dicotômica em dois fatores, que explicam conjuntamente 56,92\% da variância total: "Aspectos estruturais e organizacionais do Programa Mais Médicos" $(\alpha=0,96)$ e "A atuação dos médicos no Programa Mais Médicos" $(\alpha=0,74)$. Contempla-se, ainda, que a eficácia do PMM foi avaliada negativamente. Esses achados revelam que o instrumento é útil para avaliação do PMM, ainda escasso de instrumentos na literatura para sua avaliação, podendo ser adequadamente utilizada em estudos futuros.
\end{abstract}

Palavras-chave: avaliação de programa, saúde pública, médicos, análise fatorial

\section{Scale for Assessment of Beliefs about the Program More Doctors}

\begin{abstract}
This research aimed to create an instrument for assessing the effectiveness of the Programa Mais Médicos (PMM - Program More Doctors), verifying the perceptions of a national non-probabilistic stratified sample of 643 Brazilians. Factor analysis was performed on the scale and the PMM assessment data were analyzed using descriptive, bivariate and multivariate statistics. The Principal Components method, indicating best psychometric values and theoretical explanation, with varimax rotation, structured the 35 items of dichotomous scale into 2 factors, which explain $56.92 \%$ of the total variance: "Structural and organizational aspects of the Programa Mais Médicos" ( $\alpha=0.96)$ and "The role of doctors in the Programa Mais Médicos" $(\alpha=0.74)$. It is also contemplated that the effectiveness of PMM was negatively assessed. These findings reveal that the instrument is useful for evaluation of PMM, still short of instruments in the literature for its evaluation, and can be properly used in future studies. Keywords: program evaluation; public health; doctors; factor analysis
\end{abstract}

\section{Escala de Evaluación de las Percepciones sobre el Programa Más Médicos}

\begin{abstract}
Resumen
Esta investigación tuvo como objetivo crear un instrumento de evaluación de la eficacia del PMM, (Programa Más Médicos) tomando las percepciones de una muestra no probabilística nacional estratificada de 643 brasileños. Fue realizado análisis factorial de la escala y los datos de evaluación del PMM fueron analizados por medio de estadística descriptiva, bivariada y multivariada. Indicando mejores valores psicométricos y explicación teórica, el método Componentes Principales, con rotación varimax, estructuró los 35 ítems de la escala dicotómica en 2 factores, que explican conjuntamente 56,92\% de la varianza total: "Aspectos estructurales y organizativos del PMM" $(\alpha=0,96)$ y "Actuación de los médicos en el PMM" $(\alpha=0,74)$. Se contempla además que la eficacia del PMM fue evaluada negativamente. Esos resultados revelan que el instrumento es útil para la evaluación del PMM, todavía escaso de instrumentos en la literatura, pudiendo ser utilizado adecuadamente en futuros estudios. Palabras-clave: evaluación de programa; salud pública; médicos; análisis factorial.
\end{abstract}

\section{Introdução}

Com o objetivo de mitigar o problema da falta de médicos na Atenção Básica do Sistema único de Saúde (SUS), foram criados dois programas pelo governo federal, unificados em 2015: o "Programa de Valorização do Profissional da Atenção Básica" (PROVAB) (Portaria no 2.087, 2011) e o "Programa Mais Médicos" (PMM) (Lei 12.871, 2013). Programas recentes, escassos na literatura de avaliação de políticas públicas de saúde, fazendo-se necessária a construção de instrumentos para sua avaliação por meio da apuração das percepções dos diferentes atores sociais envolvidos (gestores, profissionais e usuários).

Em 2015, por meio da primeira edição do edital unificado do PROVAB e do PMM, foram selecionados 4.147 médicos para atuarem nos dois programas, que possuem distinção de pré-requisito para inserção. O PROVAB é exclusivo para médicos brasileiros, e o PMM é aberto a médicos brasileiros e estrangeiros, sem 
a necessidade de realização da prova de revalidação de diploma, antes obrigatória e com altas taxas de reprovação (Lei 12.871, 2013).

Em suas diretrizes teóricas, os médicos participantes do PMM devem atuar na Atenção Básica, junto a um supervisor e um tutor acadêmico. Recebem uma bolsa de R \$10.000,00 e, no caso de o participante não residir no município de trabalho, o Ministério da Saúde paga uma ajuda de custo para passagens e despesas, além de auxílio-alimentação e moradia opcionais pagos pelos municípios (Ministério da Saúde [MS], 2014).

Sua forma de implantação, objetivos e determinadas regras geram reações divididas de aceitação e reprovação do PMM pela população em geral e comunidade médica (Baião, Melo, \& Leônidas, 2014; Carvalho, 2013; Costa, Melo, Maciel, \& Santos 2014; Scremin \& Javorski, 2013). Como consequência, é despertada a necessidade de que pesquisadores se interessem pela avaliação das estratégias e programas governamentais e sobre suas políticas públicas, especialmente o PMM, observando seus erros e acertos, e oferecendo feedback (Belloni, Magalhães, \& Sousa, 2003; Melo, Alchieri, \& Araújo Neto, 2012a, 2012b, 2013; Lins, Alchieri, Araújo Neto, \& Melo, 2014). Por meio dessas pesquisas, é possível, portanto, melhorar os programas e mitigar a crença brasileira de que os serviços públicos são para pobres e que, portanto, podem ser de baixa qualidade (Albuquerque, 2002; Lins et al., 2014).

A Psicologia, dentro de uma perspectiva social, é convocada a propor metodologias que auxiliem no acompanhamento e desenvolvimento de políticas e programas, levando em conta a subjetividade de pessoas e grupos. Admite-se que o psicólogo seria um profissional indicado para tal tarefa, já que ele é preparado para construção de instrumentos de avaliação de comportamento, mediante um amplo arcabouço teórico: relações intergrupais, percepções, atitudes, diferenças entre gêneros, comportamentos individuais e grupais (Melo et al., 2012a).

Dentro da Psicologia Social, cujo objetivo principal é entender a interação humana e os processos cognitivos e afetivos envolvidos, destaca-se a percepção social, conceito fundamental na área, escolhida como objeto do presente estudo. Reconhece-se, pois, que, por meio do processo de socialização, são coletadas informações, que geram nos sujeitos impressões sobre si mesmo e seu mundo. Variáveis intervenientes que, embora se refiram a fenômenos psicológicos não acessíveis à observação direta, podem ser inferidas e integrar hipóteses empíricas, pois são acessíveis à mensuração ainda que de forma indireta (Rokeach, 1981).

A análise das percepções mostra-se de fundamental importância para o planejamento de ações e o acompanhamento das políticas públicas em saúde, intervindo também nas diretrizes e no desenho delas. Além de que, por serem propulsoras de comportamento, possibilitam a compreensão dos argumentos utilizados para justificar determinadas ações, permitindo um melhor conhecimento da realidade. Assim, a Psicologia Social aliada à Psicometria, pode auxiliar na medição do que atores sociais pensam sobre uma política pública (Candeias \& Marcondes, 1979; Lins et al., 2014; Melo et al., 2012a; Oliveira \& Albuquerque, 2008).

Reforça-se especial necessidade de conhecer a realidade do PMM em âmbito nacional, indo além de pesquisas qualitativas locais; fazendo uso de instrumentos com validação psicométrica (Albuquerque \& Melo, 2010; Cotta et al., 2005; Frekete, 2000; Lins et al., 2014). Lembra-se, pois, que a escolha do método, em especial do instrumento utilizado é uma decisão fundamental para a pesquisa de avaliação (Frekete, 2000; Furtado, 2001).

Respaldados nessa ideia, objetivou criar e apresentar evidências de validade psicométrica da Escala de Avaliação do Programa Mais Médicos a partir das percepções dos brasileiros. Pretendeu-se ainda: 1) verificar a avaliação que os brasileiros fazem da eficácia do PMM e 2) comparar os resultados da avaliação do PMM, segundo as variáveis biodemográficas dos participantes.

\section{Método}

\section{Tipo de Estudo}

O presente estudo trata-se de uma pesquisa descritiva e exploratória. Foi realizado por meio de levantamento via internet em todo o Brasil.

\section{Participantes}

A população do Brasil é de 198,7 milhões de habitantes. Para uma população desse porte, com nível de confiança de $95 \%$, e erro amostral de $5 \%$, fez-se necessário uma amostra mínima de 385 participantes. Estes foram selecionados por meio de uma amostra não probabilística dividida por quotas de região do país (com o mínimo de 77 sujeitos em cada região) (Cozby, 2003).

Após a coleta de dados, realizada durante quatro meses (novembro a fevereiro/2015), contou-se com 643 participantes, sendo $315(49 \%)$ mulheres e 328 (51\%) homens; com idade média de 31,69 anos (DP 
$=10,20)$, com variação entre 16 e 68 anos. A maioria possui ensino escolar completo $(f=584 ; 90,90 \%)$ (Tabela 1), sendo que 103 (16\%) eram médicos. São representativos de todas as regiões e estados do país: Região Norte $(f=78 ; 12,10 \%)$, Região Nordeste $(f=$ 218; 33,90\%), Região Centro-oeste ( $f=82 ; 12,80 \%$ ), Região Sudeste $(f=166 ; 25,80 \%)$ e Região Sul $(f=99$; $15,40 \%)$. Moram tanto na capital $(f=355 ; 55,20 \%)$, quanto no interior $(f=288 ; 44,80 \%)$.

Contempla-se ainda que a maioria dos participantes possui percepções diretas sobre a Estratégia Saúde da Família (ESF) e o Programa Mais Médicos, uma vez que: a maioria já foi atendida na $\operatorname{ESF}(f=466 ; 72,50 \%$ ), quase metade já foi atendida por algum médicobolsista do programa $(f=314 ; 48,80 \%)$ e a maioria conhece alguém que já foi atendido por um médico do programa $(f=499 ; 88,30 \%)$ (ver Tabela 2$)$.

\section{Instrumento}

A partir de estudo qualitativo anterior (Costa et al., 2014), foi criada e validada no presente estudo a Escala de Avaliação do Programa Mais Médicos a partir das percepções dos brasileiros, composta por 35 itens, com resposta dicotômica, distribuídos em dois fatores: 1) Aspectos estruturais e organizacionais do Programa Mais Médicos, com 24 itens, e Alpha de Crombach 0,96; e 2) A atuação dos médicos no Programa Mais Médicos, com 11 itens, alfa de Crombach 0,74 (ver processo de construção e valores psicométricos nos resultados).

\section{Procedimentos}

Considerando-se os aspectos éticos referentes a pesquisas envolvendo seres humanos, o presente estudo foi submetido à Comissão de Ética em Pesquisa da Unifor, aprovado com parecer número 1.356.319. Em seguida, foi realizado o processo de construção do instrumento de pesquisa. Após a finalização do instrumento, este foi disponibilizado na internet juntamente com o Termo de Consentimento Livre Esclarecido TCLE, por meio de uma página específica e de domínio privado. A divulgação ocorreu durante quatro meses (novembro a fevereiro/2015) por meio de redes sociais on-line (facebook) em divulgação de grupos de interesse de interesse em geral. Destaca-se ainda que foram respeitados os aspectos éticos exigidos pela Resolução $\mathrm{n}^{\circ}$ 466/12 do Conselho Nacional de Saúde.

Tabela 1

Distribuição da Amostra por Escolaridade

\begin{tabular}{lcc}
\hline Nível de escolaridade & Frequência & Porcentagem \\
\hline Analfabeto & 2 &, 3 \\
Fundamental Incompleto & 16 & 2,5 \\
Fundamental Completo & 16 & 2,5 \\
Médio Incompleto & 25 & 3,9 \\
Médio Completo & 93 & 14,5 \\
Superior Incompleto & 164 & 25,5 \\
Superior Completo & 327 & 50,9 \\
\hline
\end{tabular}

Tabela 2

Distribuição da Amostra por Proximidade do Programa

\begin{tabular}{lcc}
\hline Nível de proximidade do serviço & Frequência & Porcentagem \\
\hline Já atendido na ESF. & 466 & 72,5 \\
Nunca atendido na ESF. & 177 & 27,5 \\
Já atendido por médico do PMM. & 314 & 48,8 \\
Nunca atendido por médico do PMM. & 329 & 51,2 \\
Conhece alguém atendido por médico do PMM. & 499 & 88,3 \\
Não conhece alguém atendido por médico do PMM. & 66 & 11,7 \\
\hline
\end{tabular}




\section{Análise dos Dados}

As análises de dados foram realizadas com auxílio do pacote estatístico SPSS (Statistical Package for Social Science) for Windows versão 2.0, dividida em cinco etapas. Primeiro foi traçado o perfil da amostra por meio de estatística descritiva (frequência, porcentagem e medidas de tendência central e dispersão).

$\mathrm{Na}$ segunda etapa, com auxílio do SPSS, foram analisadas as adequações psicométricas das escalas por meio de análise fatorial exploratória utilizando metade da amostra (Principal Componentes - Principal Components - PC e Principal Eixo Fatorial - Principal Axis Factoring - PAF, com rotação oblimin, com todos os arranjos de número de fatores aceitáveis - um a quatro fatores).

Em seguida, na terceira etapa, com auxílio do pacote estatístico AMOS 18.0 foram realizadas análises fatoriais confirmatórias com a outra metade da amostra, tendo em conta a matriz de covariância e adotando o estimador ML (Máxima verossimilhança). Contempla-se que, como a escala exigia resposta obrigatória, não haviam casos omissos. Para conhecer o ajuste do modelo proposto e compará-lo com modelos alternativos, utilizou-se os seguintes indicadores: a razão do qui-quadrado pelos graus de liberdade $\left(\chi^{2} / \mathrm{gl}\right)$, onde valores menores do que cinco indicam um ajuste adequado do modelo; o goodness of fit index (GFI), o adjusted goodness of fit index (AGFI) e o comparative fit index (CFI), onde valores iguais ou superiores a 0,90 indicam ajuste bom; e o root mean square error of approximation (RMSEA), cujos valores de 0,06 ou menor indicam ajuste satisfatório, aceitando-se aqueles de até 0,10. Além desses indicadores, a diferença de qui-quadrado $\left(\Delta \chi^{2}\right)$ e o expected cross-validation index (ECVI) foram empregados para avaliar melhoras entre os modelos concorrentes testados. Valores significativos de $\Delta \chi^{2}$ e valores mais baixos de ECVI refletem um modelo com melhor ajuste (Garson, 2012).

$\mathrm{Na}$ quarta etapa, foi realizada a análise descritiva dos fatores da escala e de seus subfatores. Para tanto, todos os itens dos fatores e subfatores foram convergidos para afirmações positivas sobre o PMM. Desse modo, todas as afirmações que o participante concordasse, oferecendo avaliação "positiva" do PMM, obteve resposta "sim", e foi computado com valor 1; e todas as afirmações que o participante não concordasse, caracterizando-se avaliação "negativa" do PMM, obteve resposta "não", e foi computado com valor 0 . Em seguida, foi criado o escore total para cada fator e subfator (com pontuação máxima equivalente ao número de itens que possui): no Fator 1 ( 0 - 24 pontos) [Subfator $1.1(0-7)$; Subfator $1.2(0-4)$; Subfator 1.3 $(0-7)$; e Subfator $1.4(0-6)]$; e no Fator $2(0-11$ pontos) [Subfator $2.1(0-5)$ e Subfator $2.2(0-6)]$. Então, para melhor vizualização dessas pontuações dos fatores e subfatores, optou-se por oferecer, paralelamente, um valor equivalente padronizado em escala de 0 a 10 , gerado por meio de uma transformação linear da pontuação original por meio de regra de três simples. Por fim, as notas em escala de 0 a 10 foram interpretadas em quartis: péssimo $(0-2,5)$, ruim $(2,6-5,0)$, bom $(5,1$ $-7,5)$ e excelente $(7,6-10,0)$.

Por fim, na quinta etapa das análises, foram realizadas comparações das avaliações dos fatores e subfatores em função dos dados biodemográficos: sexo, escolaridade, já ter sido atendido em ESF, já ter sido atendido por médico bolsista do PMM, conhecer alguém atendido por médico bolsista do PMM e por categoria profissional (médicos e não médicos).

\section{Resultados e Discussão}

Os resultados são apresentados seguindo a ordem dos objetivos. Inicialmente é exposto o processo de validação psicométrica da Escala de Avaliação do Programa Mais Médicos a partir das percepções dos brasileiros e, em seguida, são apresentados os dados de avaliação do PMM pelos brasileiros.

Construção e Validação da Escala de Avaliação do Programa Mais Médicos a Partir das Percepsões dos Brasileiros

Para construção de um instrumento válido e preciso para a apuração das percepções da população sobre o PMM, buscou-se seguir o modelo de construção de instrumento baseado nos três polos elaborados por Pasquali (1999): 1) no polo teórico enfocou-se a teoria acerca do construto a ser medido pelo instrumento; 2) no polo empírico ou experimental foram definidas as etapas e técnicas de avaliação do instrumento-piloto para se ter um instrumento com boa qualidade psicométrica; e 3) no polo analítico estabeleceram-se os procedimentos de análises estatísticas dos dados na verificação da validade e a precisão do instrumento.

No primeiro momento, foram selecionados seis atributos que são representativos para a população para avaliação do PMM, emergidos a partir de estudo inicial qualitativo (Costa et al., 2014): 1) Disponibilidade de médicos no PMM; 2) Participação dos médicos estrangeiros no PMM; 3) Gestão do PMM; 4) Capacitação dos médicos do PMM; 5) Humanização no 
atendimento dos médicos do PMM; e 6) Motivação dos médicos do PMM.

A partir desses atributos, estabeleceu-se um modelo teórico inicial para construção do instrumento contendo 36 itens, que foi submetido a uma banca composta por cinco juízes (psicólogos sociais, com experiência em psicometria, avaliação de programas e conhecedores do PMM), buscando identificar os problemas no instrumento. Em seguida, contou-se com a colaboração de seis usuários da ESF e do PMM nesse processo de construção da escala, para a validação semântica e de construto dos itens. Para tanto, foi solicitado a esses usuários que expressassem o que o item questionava, a fim de verificar se ele media o que pretendia. Contempla-se que tal processo não se trata de um estudo piloto, e não tem objetivo de que os usuários respondam o instrumento, ofertando sua avaliação sobre o programa, mas sim que ele explicite a sua compreensão sobre os itens, assegurando a clareza e significado compartilhado dos itens. Assim, foi concluída a etapa teórica, ficando o instrumento a ser utilizado na aplicação da escala com 36 itens.

$\mathrm{Na}$ sequência, após coleta de dados, para o início da etapa empírico/experimental, foram feitas adequações no banco e análises iniciais na escala. Na sequência, ao início da fase analítica, foi confirmada a adequação da análise fatorial com o conjunto de 36 itens, observando-se valores ótimos do KMO $(0,98)$ e do Teste de Esfericidade de Bartlett $\left(\chi^{2}=18.218,46 ; p<0,05\right)$ (Foster, Barkus, \& Yavorsky, 2006). Destaca-se ainda que a análise de 643 sujeitos atendeu também o critério de Tabachnick e Fidel (1989) sobre o número mínimo de a amostra ser cinco vezes maior que o número de itens, pois havia uma proporção de 17,86 pessoas/item.

Em seguida, foi realizada uma primeira análise fatorial, com uso do método "Componentes Principais" (Principal Components - PC), verificando-se que todos os itens possuem coeficientes com carga fatorial acima de 0,30. Nessas primeiras análises, foram encontrados quatro fatores com eigenvalues superiores a 1, que explicavam 63,26\% da variância total. Em seguida, foram realizadas análises utilizando os dois principais métodos de análise fatorial (Principal Componentes - Principal Components - PC e Principal Eixo Fatorial - Principal Axis Factoring - PAF) com rotação oblimin (supondo a tendência de linearidade das variáveis em Psicologia), com todos os arranjos de número de fatores aceitáveis (1 a 4 fatores), observando-se que a melhor alternativa foi o arranjo apresentado pela análise de Componentes Principais, com rotação oblimin e dois fatores, que aglomerou melhor os itens no scree plot e mostrou-se mais adequada teoricamente.

Ao fim das análises, os dois fatores explicam $58,24 \%$ da variância total do construto estudado, possuem índice de consistência interna do alfa de Crombach excelente $(\alpha=0,95)$ e aglomeram 35 itens (com perda de um item - 17). Os resultados destas análises com os itens que compõem o instrumento final e suas cargas fatoriais podem ser observados na Tabela 3 .

O Fator 1, Aspectos estruturais e organizacionais do Programa Mais Médicos, é composto por 24 itens, com saturação mínima 0,53 [Item 2. Com o Programa Mais Médicos ficou mais fácil ser atendido por médico no serviço público] e máxima 0,90 [Item 15. O governo federal tem feito uma boa gestão do Programa Mais Médicos]. Possui eigenvalue 18,51, que explica 52,88\% do índice de variância, com ótimo índice de consistência interna Alpha de Crombach $(\alpha=0,96)$.

O fator é composto por itens que refletem a avaliação que a população brasileira faz sobre a resolutividade do programa como proposta para solucionar o problema da falta de médicos, avaliando ainda a participação dos médicos estrangeiros, bem como a capacitação oferecida pelo programa aos seus profissionais e sua gestão. Deste modo, para melhor apresentação de seus resultados, este fator subdivide-se teoricamente em quatro subfatores: 1.1 O PMM como proposta para a solução da falta de médicos (itens 1, 2, 7, 18, 24, 28 e 29); 1.2 Participação dos médicos estrangeiros no PMM (itens 13, 23, 27 e 34); 1.3. Capacitação dos médicos no PMM (itens 11, 12, 14, 21, 25, 32 e 33); e 1.4 "Gestão do PMM" (itens 3, 8, 9, 10, 15 e 20).

O Fator 2, A atuação dos médicos no Programa Mais Médicos, reuniu 11 itens, com saturação mínima 0,39 [Item 22. Os médicos só participam do Programa Mais Médicos porque querem receber alguma vantagem] e máxima 0,61 [Item 6. Os médicos estrangeiros do Programa Mais Médicos são atenciosos com os pacientes]. Esse fator apresentou eigenvalue 1,87 que explica 5,36\% do índice de variância, $\operatorname{com} \alpha=0,74$. Os itens que compõem este fator refletem as percepções da população sobre a atuação dos médicos bolsistas no PMM. Esse fator subdivide-se teoricamente em dois subfatores: 2.1 Humanização dos médicos do PMM (itens 4, 5, 6, 19 e 31); e 2.2 Motivação dos médicos do PMM (itens 16, 22, 26, 30, 35 e 36).

Análise Confirmatória da Escala de Avaliação do Programa Mais Médicos a Partir das Percepções dos Brasileiros

Inicialmente, realizou-se uma análise fatorial confirmatória, testando o modelo com os dois fatores 
Tabela 3

Análise Fatorial da Escala de Avaliação do Programa Mais Médicos a Partir das Percep̧ões dos Brasileiros pelo Método PC com Rotação Varimax

Itens

Fatores

1. Sou contra o "Programa Mais Médicos" porque é uma alternativa insuficiente.

Fator 1

Fator 2

2. Com o "Programa Mais Médicos" ficou mais fácil ser atendido por médico no serviço público.

3. Tem diferença entre o que diz a lei sobre o "Programa Mais Médicos" e como ele funciona na prática.

7. Ao invés de criar o "Programa Mais Médicos" o governo deveria investir na melhoria das condições de trabalho dos médicos brasileiros

8. Os recursos destinados ao "Programa Mais Médicos" são mal utilizados

0,81

0,53

0,79

9. O governo municipal tem feito uma má gestão do "Programa Mais Médicos".

0,63

10. Os gestores do "Programa Mais Médicos" são qualificados.

0,68

11. Os médicos estrangeiros do "Programa Mais Médicos" tem dificuldade com a língua

0,74 portuguesa.

12. Os médicos estrangeiros do "Programa Mais Médicos" conseguem se comunicar com os pacientes brasileiros.

13. Sou contra a vinda dos médicos formados em outros países sem diploma reconhecido no Brasil.

14. Os médicos estrangeiros do "Programa Mais Médicos" são capacitados mesmo sem diploma válido no Brasil.

15. O governo federal tem feito uma boa gestão do "Programa Mais Médicos".

0,88

0,88

18. Os médicos do "Programa Mais Médicos" vieram para trabalhar em lugares que os outros médicos não queriam trabalhar.

20. Os gestores do "Programa Mais Médicos" estão fazendo o melhor possível com os recursos que recebem

21. Os médicos que atendem no "Programa Mais Médicos" são despreparados.

23. O Brasil precisa dos médicos estrangeiros.

24. O "Programa Mais Médicos" resolveu o problema de muita gente que não tinha médico e agora tem.

25. Os médicos do "Programa Mais Médicos" são capacitados.

27. Os médicos brasileiros devem aceitar os médicos estrangeiros do "Programa Mais Médicos" aqui.

28. Sou contra o "Programa Mais Médicos" porque o problema de saúde no Brasil é $\quad 0,85$ maior do que a falta de médicos.

29. O "Programa Mais Médicos" é uma alternativa ruim para resolver o problema da falta de médicos na saúde pública.

32. Os médicos estrangeiros do "Programa Mais Médicos" são despreparados.

0,76 
Tabela 3

Análise Fatorial da Escala de Avaliação do Programa Mais Médicos a Partir das Percepções dos Brasileiros pelo Método PC com Rotação Varimax (Continuação)

Itens

Fatores

33. Os médicos estrangeiros do "Programa Mais Médicos" desconhecem os problemas e doenças da saúde pública no Brasil.

34. Sou contra a vinda de médicos estrangeiros do "Programa Mais Médicos" para o Brasil.

4. As consultas dos médicos do "Programa Mais Médicos" são muito rápidos. 0,48

5. No "Programa Mais Médicos" os médicos atuam mais de perto da comunidade. 0,41

6. Os médicos estrangeiros do "Programa Mais Médicos" são atenciosos com os 0,61 pacientes.

16. Os médicos do "Programa Mais Médicos" são interessados nos problemas dos seus Fator 1 Fator 2 pacientes.

19. Os médicos brasileiros do "Programa Mais Médicos" são atenciosos com os 0,79 0,77 pacientes.

22. Os médicos só participam do "Programa Mais Médicos" porque querem receber alguma vantagem.

26. Os médicos do "Programa Mais Médicos" querem trabalhar tanto na capital quanto nas cidades do interior.

30. Os médicos do "Programa Mais Médicos” querem melhorar a saúde no Brasil.

31. Os médicos do "Programa Mais Médicos" tem indisposição para atender os pacientes.

35. Os médicos do "Programa Mais Médicos" só se interessam em dinheiro.

36. O "Programa Mais Médicos" foi criado para que existam médicos atendendo todos os dias.

proposto na análise fatorial exploratória. O modelo proposto, denominado de modelo 1, com dois fatores, foi comparado com dois modelos alternativos: modelo 2 , unifatorial de primeira ordem; modelo 3, com 3 fatores não correlacionados. Os indicadores de ajuste para os seis modelos testados podem ser observados na Tabela 4.

Procedendo dessa forma, a estrutura fatorial resultante permitiu obter melhores indicadores de ajuste aos dados para o modelo proposto: $\chi^{2}(643)=1509,25, p$ $<0,05, \chi^{2} / g l=2,77, \mathrm{GFI}=0,87, \mathrm{AGFI}=0,85, \mathrm{CFI}=$ 0,95, RMSEA $=0,05(\mathrm{IC} 90 \%=0,05-0,06)$ e ECVI $=2,62$. Na Figura 1, é apresentada a estrutura fatorial desse modelo.
Análise Descritiva dos Fatores Da Escala de Avaliação do Programa Mais Médicos a Partir das Percepções dos Brasileiros

São apresentados nesta seção os resultados encontrados nos fatores anteriormente descritos: 1) Aspectos estruturais e organizacionais do Programa Mais Médicos; e 2) A atuação dos médicos no Programa Mais Médicos.

O Fator 1, Aspectos estruturais e organizacionais do Programa Mais Médicos, com 24 itens, apresentou uma média de respostas de $6,85(D P=7,54)$, equivalente a $2,86(D P=3,14)$ numa escala de 0 a 10 , interpretada na distribuição dos quartis como uma avaliação ruim. O subfator 1.1 O PMM como proposta para a solução da falta de médicos, com sete itens, apresentou uma 
Tabela 4

Indices de Ajuste dos Modelos Testados

\begin{tabular}{lcccccccc}
\hline Modelos & $\begin{array}{c}\chi^{2} \\
(\mathrm{gl})\end{array}$ & $\chi^{2} / \mathrm{gl}$ & GFI & AGFI & CFI & RMSEA & ECVI & $\begin{array}{c}\Delta \chi^{2} \\
(\Delta \mathrm{gl})\end{array}$ \\
\hline Modelo 1 & $1509,25(544)$ & 2,77 & 0,87 & 0,85 & 0,95 & 0,05 & 2,62 & - \\
Modelo 2 & $2674,23(527)$ & 5,07 & 0,75 & 0,71 & 0,88 & 0,08 & 4,38 & $1164,98(17)$ \\
Modelo 3 & $3451,77(560)$ & 6,16 & 0,78 & 0,75 & 0,84 & 0,09 & 5,60 & $1942,52(16)$ \\
\hline
\end{tabular}

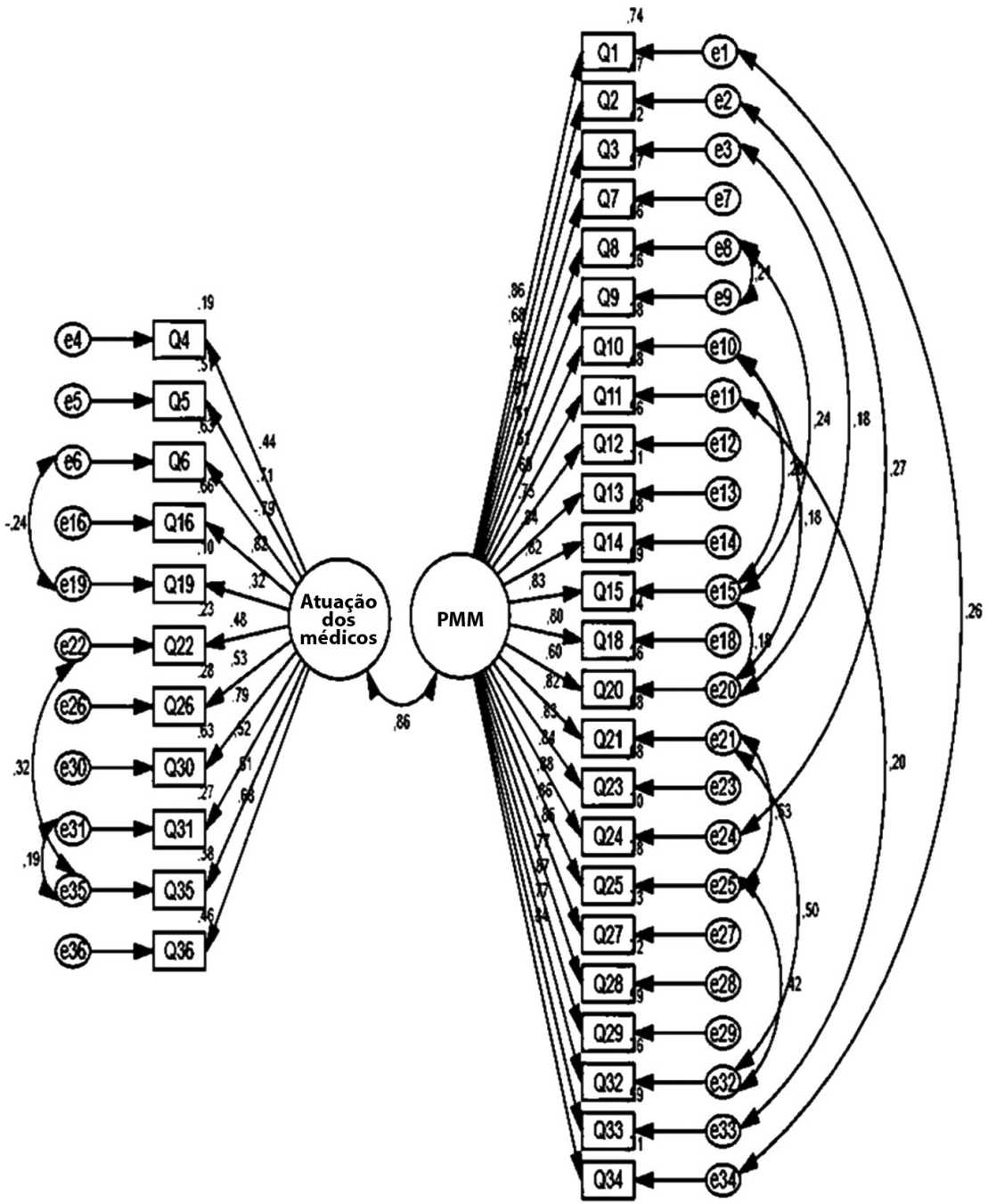

Figura 1. Estrutura fatorial do modelo com dois fatores.

média de respostas de 2,54 ( $D P=1,91)$, equivalente a 3,63 $(D P=2,72)$ na escala de 0 a 10 , interpretado como uma avaliação ruim. O subfator 1.2, Participação dos médicos estrangeiros no PMM, com quatro itens, apresentou uma média de respostas de 1,07 (DP
$=1,56)$, equivalente a $2,67(D P=3,91)$ na escala de 0 a 10 , interpretado como uma avaliação ruim. O subfator 1.3, Capacitação dos médicos no PMM, com sete itens, apresentou uma média de respostas de 2,06 (DP $=2,69)$, equivalente a $2,94(D P=3,84)$ na escala de 0 a 
10, interpretado como uma avaliação ruim. O subfator 1.4, Gestão do PMM, com seis itens, apresentou uma média de respostas de $1,19(D P=1,88)$, equivalente a $1,98(D P=3,14)$ na escala de 0 a 10 , interpretado como uma avaliação péssima.

O Fator 2, A atuação dos médicos no Programa Mais Médicos, com 11 itens, apresentou uma média de respostas de 5,37 $(D P=2,84)$, equivalente a 4,88 $(D P=$ $2,58)$ numa escala de 0 a 10 , interpretada na distribuição dos quartis como uma avaliação ruim. O subfator 2.1, Humanização dos médicos do PMM, com cinco itens, apresentou uma média de respostas de 2,75 (DP $=1,03)$, equivalente a $5,51(D P=2,07)$ na escala de 0 a 10 , interpretada como uma avaliação boa. O subfator 2.2, Motivação dos médicos do PMM, com seis itens, apresentou uma média de respostas de 2,62 $(D P=2,12)$, equivalente a 4,36 $(D P=3,54)$ na escala de 0 a 10 , interpretada como uma avaliação ruim (Tabela 5).

É possível verificar ainda que nas atribuições das notas de avaliação houve diferenças percebidas pelas comparações realizadas a partir das variáveis biodemográficas. A seguir são apresentadas apenas as avaliações que se destacaram pela existência de diferenças estatisticamente significativas. Para tanto, destaca-se que todas essas variáveis se apresentaram não normais a partir do teste de Shapiro-Wilk: fator $1(W=0,74 ; p<0,01)$, subfator $1.1(W=0,77 ; p<0,01)$, subfator $1.2(W=$ $0,67$; $p<0,01)$, subfator 1.3 ( $W=0,73$; $p<0,01$ ), subfator $1.4(W=0,67 ; p<0,01)$, fator $2(W=0,94 ; p<$ $0,01)$, subfator $2.1(0,89 ; p<0,01)$, subfator 2.2 ( $W=$ $0,89 ; p<0,01)$. Portanto, foram utilizados os testes não paramétricos Mann-Whitney e Kruskal-Wallis.

Verificou-se que houve diferenças estatisticamente significativas nas comparações por escolaridade, em todos os fatores e subfatores da escala. Constatou-se que as piores avaliações foram feitas pelos participantes com índices de escolaridade mais baixo (fundamental incompleto e completo); e as melhores avaliações pelos participantes com maiores índices de escolaridade (superior incompleto e completo):

Fator 1. $\left[\chi^{2}(6, N=643)=62,00, p<0,05\right]$; Subfator $1.1\left[\chi^{2}(6, N=643)=58,51, p<0,05\right]$; Subfator $1.2\left[\chi^{2}(6, N=643)=50,21, p<0,05\right]$; Subfator $1.3\left[\chi^{2}\right.$ $(6, N=643)=70,91, p<0,05]$; Subfator $1.4\left[\chi^{2}(6, N\right.$ $=643)=24,65, p<0,05]$; Fator 2. $\left[\chi^{2}(6, N=643)=\right.$ 68,32, $p<0,05]$; Subfator $2.1\left[\chi^{2}(6, N=643)=32,77\right.$, $p<0,05]$; Subfator $2.2\left[\chi^{2}(6, N=643)=71,41, p<\right.$ $0,05]$. Destaca-se que, apesar das pessoas com maior escolaridade avaliarem melhor o PMM, estas também atribuem avaliações negativas ao programa na maioria dos quesitos, com exceção do fator 2. A atuação dos médicos no Programa Mais Médicos.

Além da escolaridade, realizaram-se comparações entre médicos e não médicos, verificando-se diferenças estatisticamente significativas em quase todos os fatores e subfatores, onde os médicos atribuíram pior avaliação do PMM: Fator 1. $(U=21461,00, p<0,05)$; Subfator $1.1(U=20847,50, p<0,05)$; Subfator $1.2(U$ $=20760,50, p<0,05)$; Subfator $1.3(U=22006,00, p<$ 0,05); Subfator $1.4(U=20797,50, p<0,05)$; Fator 2 . $(U=22962,00, p<0,05)$; Subfator $2.2(U=22855,00$, $p<0,05)$. Contempla-se, novamente, que, apesar dos não médicos avaliarem melhor o PMM, estas também atribuem avaliações negativas ao programa na maioria dos quesitos, com exceção do fator 2. A atuação dos médicos no Programa Mais Médicos.

Também foram realizadas comparações entre os usuários e não usuários da ESF, verificando-se que

Tabela 5

Avaliacões dos Fatores e Subfatores

\begin{tabular}{lcc}
\hline \multirow{2}{*}{ Fatores e subfatores } & \multicolumn{2}{c}{ Dados estatísticos em escala de 0 a 10 } \\
\cline { 2 - 3 } & Média $(D P)$ & Avaliação \\
\hline Fator 1 & $4,88(2,58)$ & Ruim \\
Subfator 1.1 & $3,63(2,72)$ & Ruim \\
Subfator 1.2 & $2,67(3,91)$ & Ruim \\
Subfator 1.3 & $2,94(3,84)$ & Ruim \\
Subfator 1.4 & $1,98(3,14)$ & Péssimo \\
Fator 2 & $4,88(2,58)$ & Ruim \\
Subfator 2.1 & $5,51(2,07)$ & Bom \\
Subfator 2.2 & $4,36(3,54)$ & Ruim \\
\hline
\end{tabular}


houve diferenças estatisticamente significativas entre os participantes devido à proximidade com o serviço, em todos os fatores e subfatores: Fator 1. $(U=31349,50$, $p<0,05)$; Subfator $1.1(U=29931,50, p<0,05)$; Subfator $1.2(U=31720,00, p<0,05)$; Subfator $1.3(U=$ 31649,50, $p<0,05)$; Subfator $1.4(U=32772,50, p<$ $0,05)$; Fator 2. $(U=33930,00, p<0,05)$; Subfator 2.1 $(U=35235,50, p<0,05)$; Subfator $2.2(U=34383,00$, $p<0,05)$. Contempla-se, novamente, que a maioria dos fatores e subfatores foram avaliados negativamente por ambos os grupos com exceção do fator 2 . A atuação dos médicos no Programa Mais Médicos e seus subfatores.

Por fim, observou-se que nos dois fatores e em quase todos os subfatores (exceto 1.4 Gestão do PMM) houve diferenças estatisticamente significativas entre as regiões do país, observando-se que as piores avaliações foram feitas pelos participantes que residem nas regiões sudeste e sul (sendo que o fator 1 e seus subfatores foram pior avaliados na região sudeste; e o fator 2 na região sul) e as melhores avaliações foram dadas pelos participantes com residem nas regiões centro-oeste, nordeste e norte (sendo que em todos os fatores e subfatores foram melhor avaliados na região centro-oeste):

Fator 1. $\left[\chi^{2}(5, N=643)=3110,50, p<0,05\right]$; Subfator $1.1\left[\chi^{2}(5, N=643)=3015,00, p<0,05\right]$; Subfator $1.2\left[\chi^{2}(5, N=643)=3158,50, p<0,05\right]$; Subfator $1.3\left[\chi^{2}(5, N=643)=3235,50, p<0,05\right]$; Fator 2. $\left[\chi^{2}(5, N=643)=3170,50, p<0,05\right]$; Subfator 2.2 $\left[\chi^{2}(5, N=643)=3030,00, p<0,05\right]$. Contempla-se, novamente, que apesar de haver melhores avaliações na região centro-oeste, esta também avaliou negativamente o PMM em todo o fator 1, havendo avaliações positivas apenas no fator 2. A atuação dos médicos no Programa Mais Médicos.

\section{Discussão}

O estudo apresenta evidências satisfatórias de validade fatorial e consistência interna de uma nova medida acerca das percepções da população sobre o Programa Mais Médicos. A análise fatorial confirmatória da medida apontou a adequação da estrutura fatorial proposta, embora alguns índices de ajuste obtidos sejam limítrofes ao que é comumente referido na literatura como desejável (Garson, 2012). Reconhece-se aqui uma medida que representa não apenas o saber científico sobre o tema, mas também as crenças compartilhadas socialmente, por isso a escala foi construída a partir dos discursos de pesquisa qualitativa anterior (Costa et al., 2014), e contemplou, na presente pesquisa, amostra de diferentes extratos sociais e distintos níveis de escolarização. Representando, assim, uma contribuição para suprir a necessidade de ferramentas de avaliação continuada de programas sociais (Lins et al., 2014; Melo et al., 2012a).

A avaliação geral do programa a partir dos resultados deste trabalho foi ruim, podendo-se inferir que o PMM não foi reconhecido pela amostra de usuários como uma solução suficiente para sanar a lacuna da falta de médicos no SUS. Os resultados mostram que os participantes avaliaram de forma ruim os aspectos estruturais e organizacionais do Programa Mais Médi$\cos$ (Fator 1). Evidencia uma tendência de insatisfação com alguns aspectos do programa, em consonância com a classe médica, que desde o princípio critica-o na mídia e nas redes sociais (Baião et al., 2014; Costa et al., 2014).

Entre os subfatores do fator 1, O PMM como proposta para a solução da falta de médicos (subfator 1.1) também foi avaliado como ruim, mostrando que, apesar de a atração de médicos para a Atenção Básica ser o principal objetivo do PMM e esse ser o principal benefício apontado pelos seus defensores (Garcia et al., 2014), para os participantes da presente pesquisa o PMM não é suficiente para sanar esta lacuna. A Participação dos médicos estrangeiros no PMM (subfator 1.2) também foi avaliada como ruim, corroborando com o mal-estar gerado entre a classe médica brasileira com a vinda dos profissionais estrangeiros (Scremin \& Javorski, 2013). A Capacitação dos médicos no PMM (subfator 1.3) foi ainda avaliado como ruim, corroborando com a literatura que aponta a falta de critérios técnicos de capacitação na seleção dos profissionais (Lins et al., 2014). A Gestão do PMM (subfator 1.4) foi avaliada como péssima, a pior da pesquisa, seguindo a tendência dos problemas existentes na gestão pública de saúde (Melo et al., 2012a; 2013).

A atuação dos médicos no Programa Mais Médicos (Fator 2), foi avaliada como ruim. Apresentou, todavia, uma nota superior à atribuída ao fator 1 , evidenciando que, para os participantes da presente pesquisa, o PMM é pior avaliado do que a atuação de seus médicos. Entre os subfatores do fator 2 , a Humanização dos médicos do PMM (subfator 2.1) foi a única avaliação boa, corroborando com a literatura que mostra que um dos fatores positivos dos serviços em ESF, em consonância com seus ideais de vínculo equipe-comunidade, é a humanização no atendimento (Albuquerque \& Melo, 2010). Já a Motivação dos médicos do PMM (subfator 2.2) foi avaliada como ruim, já que o trabalho em Estratégia de 
Saúde da Família é visto com pouco prestígio e status social na classe médica, sendo, consequentemente, evitado pelos médicos (Carvalho, 2013). Outro fator que pode corroborar é o fato do médico trabalhar sem estrutura e sem estabilidade profissional em grande parte dos postos de saúde (Albuquerque \& Melo, 2010; Melo et al., 2012a, 2012b, 2013).

Contempla-se ainda um resultado merece especial reflexão. Observou-se que na amostra da presente pesquisa, as pessoas com menos escolaridade ter avaliado o PMM de forma pior do que aqueles que possuem mais escolaridade. Reconhece-se, pois, que a população de menor escolaridade é a maior usuária dos serviços públicos de saúde e a que mais se beneficia com um programa de atração de médicos e, consequentemente, quem melhor o avaliaria. Diante da constatação de suas piores avaliações, pode-se inferir que a amostra da pesquisa apresenta uma tendência da população em ser mais exigente com os resultados dos programas públicos; ou que estes são os sujeitos mais enviesados pela mídia, que predominantemente critica o programa e forma opiniões de oposição ao governo (Albuquerque \& Melo, 2010; Melo et al., 2012a).

Contempla-se ainda que alguns resultados, como a pior avaliação do PMM pelos médicos, confirmam a tendência vista na mídia e redes sociais, que mostram que estes profissionais são, em sua maioria, contrários ao programa (Carvalho, 2013; Costa et al., 2014).

Constatou-se também que o programa foi pior avaliado pelos participantes que residem nas regiões Sudeste e Sul. Acredita-se que este fator pode ter acontecido devido à rejeição conhecida nessas regiões ao Partido dos Trabalhadores (PT), governo responsável pela criação e implantação do programa (Carvalho, 2013; Costa et al., 2014).

\section{Considerações Finais}

O Programa Mais Médicos causou opiniões divergentes, contrárias e a favor, desde o início, sendo alvo de críticas, especulações da mídia, instrumento de manobra política e com opiniões divergentes entre a população, profissionais e estudiosos. Diante da necessidade de realizar uma primeira avaliação geral do programa, os resultados desta pesquisa servem como retorno para gestores e profissionais do PMM, pois oferece uma opção de instrumento com parâmetros psicométricos adequados que pode servir de suporte para avaliação continua do programa. Além disso, os resultados deste estudo indicam como aqueles que mais conhecem na prática os serviços de saúde, os usuários e a população brasileira em geral, tendem a avaliar o programa.

Conclui-se que o PMM é um programa que apresenta avaliações negativas internas (entre seus profissionais) e externas (entre a população). Reconhece-se sua importância como uma medida provisória eficaz para atrair médicos. Sugere-se, todavia, que sejam tomadas medidas que vão além da criação do PMM. Faz-se necessária a melhoria das estruturas do sistema e das condições de atendimento e criação de estratégias de atração e manutenção de profissionais na Atenção Básica, por meio de planos de cargos e carreiras. Torna-se, portanto, emergencial a oferta de melhores condições para o atendimento dos usuários no serviço de saúde pública.

Reconhece-se a limitação do estudo por ter sido realizado via internet, onde muitas regiões do país têm pouco acesso. Decisão metodológica tomada reconhecendo o custo benefício vantajoso para atingir a finalidade de uma pesquisa a nível nacional que abrangesse participantes de diferentes características biodemográficas (sexo, escolaridade, nível socioeconômico e região do país).

Vê-se também a necessidade de realização de pesquisa com amostra probabilística representativa da população brasileira. Reforça-se ainda a importância da manutenção continuada e ampliada de pesquisas de avaliação do PMM, tendo como foco outros atores sociais envolvidos no programa (especialmente os gestores, tutores e profissionais médicos bolsistas do programa), inclusive com a criação de novos instrumentos, mais aperfeiçoados e voltados para esses outros públicos-alvo, a partir da perspectiva de diferentes linhas de pesquisas, em especial, por psicólogos, profissionais capacitados tecnicamente para tal tarefa. Sugere-se ainda a necessidade de criação de mais instrumentos com parâmetros psicométricos adequados para avaliação do PMM com os profissionais.

\section{Referências}

Albuquerque, F. J. B. (2002). Social psychology and rural life in Brazil. Psicologia: Teoria e Pesquisa, Brasília, 18(1), 37-42. Recuperado de http://www.scielo. br/pdf/ptp/v18n1/a05v18n1.pdf

Albuquerque, F. J. B., \& Melo, C. F. (2010). Avaliação dos serviços públicos de saúde em duas capitais nordestinas do Brasil. Psicologia: Teoria e Pesquisa, 26(2), 323-330. Recuperado de http:/ /www.scielo. br/pdf/ptp/v26n2/a14v26n2.pdf 
Baião, D. C., Melo, C. L, \& Leônidas, S. R. (2014). Avaliação do programa mais médicos: Uma revisão da literatura. Em Anais do XIV Encontro de Pós-graduação e Pesquisa da UNIFOR. Anais do Anais do XIV Encontro de Pós-graduação e Pesquisa da UNIFOR, Fortaleza, Ceará, Brasil. Recuperado de https:// sistemas.unifor.br/encontroscientificos/2015/ encontro/918

Belloni, I., Magalhães, H., \& Sousa, L. C. (2003). Metodologia de avaliação em políticas públicas ( $3^{\mathrm{a}}$ ed.). São Paulo: Cortez.

Candeias, N. M. F., \& Marcondes, R. S. (1979). Diagnóstico em educação em saúde: Um modelo para analisar as relações entre atitudes e práticas na área da saúde pública. Revista de Saúde Pública, 13(2), 63-68. Recuperado de http://www. scielo.br/scielo.php?script $=$ sci_arttext\&pid $=\mathrm{S} 003$ 489101979000200002\&lng=pt\&tlng=pt.10.1590/ S0034-89101979000200002

Carvalho, M. S. (2013). Programa de valorização dos profissionais da atenção básica: um Olhar implicado sobre sua implantação (Dissertação de mestrado). Brasília. Recuperado de http:/ / repositorio.unb.br/bitstream/ 10482/13660/1/2013_M\%C3\%B4nicaSampaiod eCarvalho.pdf

Costa, M. C., Melo, C. F., Maciel, R. H., \& Santos, J. B. F. (2014). A representação social dos cearenses sobre o programa mais médicos. Em Anais do XX Encontro de Iniciaşão à Pesquisa - Universidade de Fortaleza, Fortaleza. Recuperado de https://sistemas.unifor. br/encontroscientificos/2015/encontro/918

Cotta, R. M. M., Marques, E. S, Maia, T. M., Azeredo, C. M., Schott, M., Franceschini, S. C. C., \& Priore, S. E. (2005). A satisfação dos usuários do Programa de Saúde da Família, avaliando o cuidado. Scientia Medica. 15(4), 227-234. Recuperado de http://pesquisa.bvs.br/brasil/resource/pt/lil-445260

Cozby, P. C. (2003). Métodos de pesquisa em ciências do comportamento. São Paulo, SP: Atlas.

Foster, J. J., Barkus, E., \& Yavorsky, C. (2006). Understanding and using advanced statistics. Londres, Inglaterra: SAGE publications. Recuperado de https://port. rl.talis.com/items/5AC0346B-1253-5BF7-7B6C39D48B5CF900.html

Frekete, M. C. (2000). Estudo da acessibilidade na avaliação dos serviços de saúde. Manuscrito não publicado. Projeto GERUS.
Furtado, J. P. (2001). Um método construtivista para a avaliação em saúde. Ciência e Saúde Coletiva. 6(1), 165-181. Recuperado de http:// www.scielo.br/scielo.php?script $=$ sci_arttext\&pi$\mathrm{d}=$ S1413-81232001000100014

Garcia, B., Rosa, L., \& Tavares, R. (2014). Projeto Mais Médicos para o Brasil: Apresentação do programa e evidências acerca de seu sucesso. Informações Fundação Instituto de Pesquisas Econômicas (FIME). Recuperado de http://ptdocz.com/doc/31640/ projeto-mais-m $\% \mathrm{C} 3 \% \mathrm{~A} 9$ dicos-para-o-brasil-apresenta $\% \mathrm{C} 3 \% \mathrm{~A} 7 \% \mathrm{C} 3 \% \mathrm{~A} 3 \mathrm{o}-\mathrm{do}$

Garson, G. D. (2012). Structural Equation Modeling. Asheboro, NC: Statistical.

Lei 12.871, de 22 de outubro de 2013. Institui o "Programa mais médicos" e o "Projeto Mais médicos para o Brasil. Recuperado de http:/ /www.planalto.gov.br/ ccivil_03/_ato2011-2014/2013/Lei/L12871.htm

Lins, C. F. M., Alchieri, J. C., Araújo Neto, J. L., \& Melo, F. A. F. (2014) Desenvolvimento de instrumentais para avaliação da estratégia saúde da família em Natal. Psicol. Reflex. Crit, 27(2), 219-227. doi: 10.1590/1678-7153.201427202

Melo, C. F., Alchieri, J. C., \& Araújo Neto, J. L. (2012a). Assessment of the family health strategy through the beliefs of its managers. Revista Enfermagem UFPE on line, 6(2), 274-278. Recuperado de http:/ / www.revista.ufpe.br/revistaenfermagem/index. $\mathrm{php} /$ revista/article/viewArticle/2107

Melo, C. F., Alchieri, J. C., \& Araújo Neto, J. L. (2012b). Sistema Único de Saúde: uma avaliação realizada em Natal, Rio Grande do Norte, Brasil. Psico-USF, 17(1), 63-72. doi: 10.1590/ S1413-82712012000100008

Melo, C. F., Alchieri, J. C., \& Araújo Neto, J. L. (2013). Evaluation of the technical and professional profile of managers of the Brazilian Unified Health System (SUS). Revista de Enfermagem UFPE On Line, 7(7), 4670-4680. Recuperado de http://www.revista.ufpe.br/revistaenfermagem/index.php/revista/ article/viewArticle/4151

Ministério da Saúde [MS] (2014). Mais Médicos supera meta com mais 3,5 mil profissionais. Recuperado de http:/ / www.brasil.gov.br/saude/2014/04/mais-medicos-supera-meta-com-mais-3-5-mil-profissionais

Psico-USF, Bragança Paulista, v. 22, n. 3, p. 501-513, set./dez. 2017 
Ministério da Saúde [MS] (2015). Mais Médicos vai beneficiar cerca de 63 milhöes de brasileiros em 2015. Recuperado de http://www.brasil.gov.br/as ude/2015/02/mais-medicos-vai-beneficiar-cerca-de-63milhoes-de-brasileiros-em-2015

Oliveira, S. F., \& Albuquerque, F. J. B. (2008). Avaliação do Programa Saúde da Família a partir dos seus prestadores de serviço. Psicologia e Sociedade (Impresso), 20(2), 76-86. Recuperado de http://www. scielo.br/pdf/psoc/v20n2/a11v20n2.pdf

Pasquali, L. (1999). Histórico dos instrumentos psicológicos. Em L. Pasquali. (Ed.), Instrumentos psicológicos: Manual prático de elaboração (pp. 1-12). Brasília: IBAPP. Recuperado de http://pt.scribd.com/ doc/64819811/LUIS-PASQUALI\#scribd

Portaria interministerial $n^{\circ} 2.087$, de $1^{\circ}$ de setembro de 2011. Institui o Programa de Valorização do Profissional da Atenção Básica. Recuperado de http:// bvsms.saude.gov.br/bvs/saudelegis/gm/2011/ pri2087_01_09_2011.html
Rokeach, M. (1981) Crenças, atitudes e valores. Rio de Janeiro: Ed. Interciência.

Scremin, L., \& Javorski, E. (2013). O enquadramento das noticias sobre os estrangeiros do Programa Mais Médicos. Em 9o Ciclo de Debates sobre Jornalismo UniBrasil. Curitiba, Paraná, Brasil. Recuperado de http:// www.academia.edu/5922404/O_ENQUADRAMENTO_DAS_NOT\%C3\%8DCIAS_SOBRE_ OS_ESTRANGEIROS_DO_PROGRAMA_ MAIS_M $\%$ C3\%89DICOS

Tabachnick, B. G., \& Fidell, L. S. (2013). Using multivariate statistics ( $\left.6^{\mathrm{a}} \mathrm{ed}\right)$. Boston, EUA: Allyn and Bacon. Recuperado de http://www.unt.edu/rss/ class/Jon/ ResourcesWkshp/2001_TabachnickFidell_Ch4.pdf

Recebido em: 15/01/2016

Reformulado em: 02/05/2016; 21/06/2016; 11/07/2016 Aprovado em: 09/08/2016

Sobre as autoras:

Cynthia de Freitas Melo é psicóloga (2007), mestre em Psicologia Social pela Universidade Federal da Paraíba (2009), doutora em Psicologia pela Universidade Federal do Rio Grande do Norte (2013), professora do Programa de Pós-Graduação em Psicologia da Universidade de Fortaleza (UNIFOR) e coordenadora do Laboratório de Estudos e Práticas em Psicologia e Saúde (LEPP-Saúde).

E-mail:cf.melo@yahoo.com.br

Mariana Carvalho Costa é psicóloga pela Universidade de Fortaleza.

E-mail: carvalho.costa_mariana@hotmail.com

Bárbara Jéssyca Magalhães é psicóloga (2017) e mestranda em Psicologia pela Universidade de Fortaleza. E-mail: bajessyca@gmail.com

Contato com as autoras:

Av. Washington Soares, 1321, Edson Queiroz

Fortaleza-CE, Brasil

CEP: 60.811-905

Psico-USF, Bragança Paulista, v. 22, n. 3, p. 501-513, set./dez. 2017 
